\section{Einblicke in den europäischen Diakonie-Dachverband} Eurodiaconia

Roland Luri

Mit dieser wichtigen christlichen Botschaft leitet die Eurodiaconia in Bruxelles den Jahresbericht 2018 ein: ${ }^{1}$ "Wahrlich, ich sage Euch: Wiefern ihr es einem dieser meiner geringsten Brüder getan habt, habt ihr es mir getan» (Mt 25,40). Dieser kurze Evangeliumstext steht in Verbindung mit den Debatten und Anlässen, die anschliessend im Bericht näher vorgestellt werden. Sie zeigen, dass der Europäische Dachverband Eurodiaconia mit seinen Möglichkeiten versucht, das anspruchsvolle und verpflichtende Evangeliumswort praktisch umzusetzen.

Das europäische Netzwerk Eurodiaconia ist ein Dachverband von 49 christlichen sozialen Institutionen, Diakoniewerken und nationalen Kirchenvertretungen der Diakonie. Fünf Schweizer Werke (Evang.-ref. Diakonenschaft Greifensee, HEKS, Schweizer Diakonieverein Nidelbad, Sozialwerk Pfarrer Sieber, Stadtmission Zürich) sind zusammen mit der Konferenz Diakonie Schweiz Umbrella-Mitglied bei der Eurodiaconia.

Im Jahresbericht wird die Vision der Eurodiaconia wie folgt beschrieben: «Durch unseren christlichen Glauben angetrieben, haben wir eine Vision von Europa, in dem jeder Mensch für seinen inhärenten Wert und seine Würde von Gott geschätzt wird und in dem unsere Gesellschaften soziale Gerechtigkeit für alle Menschen garantieren, einschliesslich der am stärksten gefährdeten und marginalisierten Menschen».

Die Generalsekretärin der Eurodiaconia Heather Roy und ihre FachmitarbeiterInnen sind vor allem in drei Tätigkeitsfeldern aktiv:

1 Jahresbericht von Eurodiaconia: https://www.eurodiaconia.org/2019/03/our-2018annual-report-is-out-now-new-format-and-infographics-to-focus-on-impact/ (abgerufen am 11.04.19).

Jahrbuch Diakonie Schweiz 3 (2019) - ISSN 2504-3994

Dieser Text ist lizenziert unter einer Creative Commons Namensnennung 4.0 International Lizenz (CC BY 4.0): (https://creativecommons.org/licenses/by/4.0/).
- $\quad$ Armut und soziale Ausgrenzung

- Sozial- und Gesundheitsdienstleistungen

Ausgleich von Sozial- und Wirtschaftspolitik

Den Hauptteil der Finanzen von Eurodiaconia trägt der Fond «European Commission PROGRESS Grant», die Mitgliederbeiträge steuern gerade einmal ein Viertel zur Finanzierung bei. Aus diesem Grunde pflegt die Eurodiaconia einen intensiven Austausch und macht gezielte Lobbyarbeit bei den Verantwortlichen und Arbeitsgruppen der EU-Kommission, die sich mit den sozialen Fragen, Herausforderungen und sozialen Investitionen in Europa beschäftigen.

An der Generalversammlung vom 13.-15. März 2019 in Edinburgh wurde die Erklärung «Telling truth, changing lives» verabschiedet ${ }^{2}$. An dieser Versammlung gab es auch ein Plenumsgespräch mit Statements zum Thema «Truth and how that relates to diaconia» und entsprechende Workshopangebote. Anne Brigitte Pessi von der Universität Helsinki hielt einen Vortrag zu «The Power of compassion and it's relation to truth».

Laut dem Dachverband soll die Erklärung einen Beitrag zur Wahrheitsfindung angesichts wachsender Unsicherheit in Europa leisten. Inmitten einer grösser werdenden unübersichtlichen Vielfalt von Wahrheiten sei es wichtig, dass die Diakonie als ehrlicher und vertrauenswürdiger Partner wahrgenommen werde, so die Erklärung. Aufgrund des immerwährenden Stroms von Fake News durch die sozialen Netzwerke und Medien entsteht eine grosse Gefahr: Die Menschen wenden sich nicht nur gegenüber den Medien, sondern auch gegenüber den zivilgesellschaftlichen und politischen Institutionen ab. Sie klinken sich auch aus dem sozialen Diskurs aus und gehen populistischen Rhetoriken auf den Leim, die das politische System und auch die Gesellschaft untergraben.

2 Erklärung zugänglich unter: https://www.eurodiaconia.org/2019/03/agm-declaration-2019-telling-truth-changing-lives/ (abgerufen am 11.04.19).

Jahrbuch Diakonie Schweiz 3 (2019) http://dx.doi.org/10.22018/JDS.2019.10 
Die 49 diakonischen Einrichtungen und Organisationen aus Europa verpflichten sich in der Erklärung zu folgendem Statement: «Wir haben keine Angst, auch unbequeme Wahrheiten auszusprechen. Dies wird aber mit Respekt und Mitgefühl getan». Weiter steht im Erklärungstext, dass das besondere Augenmerk der Eurodiaconia der Verteidigung der Menschenrechte Schwacher und Benachteiligter gelte. Man strebe nach sozialer Gerechtigkeit und stehe mutig für die Schwächsten ein. Dabei müsse man den Machthabenden wahrheitsgetreu auch lokale Realitäten vermitteln.

Auch in der Schweiz sollen Kirchen und Werke die faktische Realität im Sozialen sehen, beurteilen und gegebenenfalls Stellung beziehen. Kirchen und Werke haben den Auftrag und die Verpflichtung, sich durch kompetente Personen ins Politische einzumischen. Nämlich dort, wo sie soziale Missstände und Ungerechtigkeiten feststellen, dürfen und sollen sie sich mit ihrer Stimme äussern. Das ist und wird auch in Zukunft die prophetische Aufgabe der christlichen Institutionen sein!

Im 2018 veröffentlichte die Eurodiaconia eine eigene Untersuchung zur Lebenssituation von Roma in Europa. Der Bericht macht deutlich, dass die gesundheitliche Versorgung, die schlechte Wohnsituation und die Armut der Roma bekämpft und verbessert werden müssen. Mit dieser Veröffentlichung möchte Eurodiaconia der Stigmatisierung der Roma entgegenwirken und Regierungen sowie die Zivilgesellschaft zur Unterstützung der Roma anregen. Es werden auch mehrere konkrete Projekte aus Serbien, Rumänien, Tschechien, Norwegen, Schweden und Finnland vorgestellt. Bereits zuvor hat Eurodiaconia mit einem Positionspapier eine verstärkte Einbeziehung der Roma in die gesellschaftliche Entwicklung gefordert.

Das der Untersuchungsbericht auch Roma-Projekte in den nordischen Ländern vorstellt, weist darauf hin, dass die Roma wegen ihrer schlechten Situation in den Herkunftsländern und trotz geringen Chancen auf eine Aufenthaltsbewilligung vor allem in nordische Länder migrieren. Auch in der Schweiz stellen zahlreiche Roma aus osteuropäischen Ländern Asylgesuche, auf die juristisch fast nie eingetreten wird. Zuweilen sind in Schweizer Städten Bettler und Musikanten dieser Ethnie aktiv und wahrnehmbar. Sie bleiben für die erlaubte Aufenthaltszeit in der Schweiz und besuchen die unentgeltlichen bzw. günstigen Essens- und Schlafangebote der diakonischen Werke. HEKS Osteuropa fördert die soziale, wirtschaftliche und gesellschaftliche Integration der Roma-Bevölkerung. Es unterstützt Projekte in Kosovo, Rumänien, Serbien und Ungarn, die eine Verbesserung der Lebenssituation und sozialen Integration der Roma zum Ziel haben.

Am 3.-4. Mai 2018 fand ein Migration Network Meeting in Rom statt. Gastgeber war die Heilsarmee Italien. Euroadiaconia möchte durch das Treffen mit Delegierten aus anderen europäischen Ländern einen geschlechterspezifischen Umgang zur Migration aus diakonischer Perspektive fördern. 2017 wurde festgestellt, dass die migrierenden Mädchen und Frauen 52\% ausmachen. Diese Zahl weist auf besondere Herausforderungen wie spezifische Rechtsberatung für Frauen, psychologische Beratung und auch frauenspezifische Sprachtrainings hin. Eurodiaconia plant die Erstellung von Migrationsleitlinien für einen geschlechtersensiblen $\mathrm{Zu}$ gang zu Unterstützung und Beratung. In Kürze werden die Leitlinien auf der Internetseite von Eurodiaconia (www.eurodiaconia.org) bereitgestellt.

Die Publikation «Eurodiaconia's Guidelines for the Integration of Migrant Women» kann den schweizerischen Werken und Kirchen wichtige Hinweise geben, wie der geschlechterspezifische Zugang zu Unterstützungsangeboten gefördert und nachhaltig sichergestellt werden kann.

Autor:

Roland Luzi, Leiter der IG-Werke, Vorsitzender der AG Kirchen und Werke der Konferenz Diakonie Schweiz.
Jahrbuch Diakonie Schweiz 3 (2019) - ISSN 2504-3994

CC by 4.0
Jahrbuch Diakonie Schweiz 3 (2019) http://dx.doi.org/10.22018/JDS.2019.10 